THE KURUME MEDICAL JOURNAL Vol.2, No. 3, 1955

\title{
UEBER DIE INNERVATION DER NEBENNIERE VON MEERSGHWEINGHEN
}

\author{
KAZUHIKO IWAKI \\ Institut für Anatomie, (Vorstand: Prof. Y. Takesige) Medizinischen Fakultït, \\ Universitäit zu Kurume, Japan
}

(Der Hauptinhalt der vorliegenden Arbeit wurde in der 11. Kyushu-lokalversammlung der anatomischen Gesellschaft in Japan veröffentlicht.)

Seit Takamine im Jahre 1901 Adrenalin, das Nebennierenmarkhormon, entdeckte, ist die Nebenniere, insbesondere das Mark, ein Lieblingskind der Zeit geworden. Seitdem aber Selye im Jahre 1938 den Begriff des Adaptationsyndrom vertrat, ist auch die Nebennierenrinde zuerst innersekretorisch mit grossem Interesse verfolgt worden. Die Nebenniere besteht aus zwei sowohl genetisch als auch funktionell ganz unterschiedlichen Teilen, der Rinde und dem Mark, und ihr endokriner Vorgang hat es innigst mit den autonomen Nervensystemen zu tun. Es wäre deswegen sehr interessant, ihre Innervation in bezug auf die Funktion der Nebenniere zu ergründen.

Ueber die Innervation der. Nebenniere finden sich schon seit Dogiel $(1894)^{(2)}$, zahlreiche Arbeiten von Renner (1914)(15), Hoshi $(1926)^{(6)}$, Kolossow $(1930)^{(10)}$, Hirt $(1930)^{(5)}$, Alpert $(1931)^{(1)}$, Kura (1932)(11), Pines und Karowtschatowa $(1932)^{(14)}$, Stöhr $(1935)^{(19)}$, Swinyard $(1937)^{(20)}$, Sato $(1951)^{(17)}$, Watanabe und Mimura $(1953)^{(21)}$, u. a. Aber wegen der Schwierigkeiten der Färbung sowohl wie der beträchtlichen Feinheit der Endigungsnervenfasern, und sogar wegen der Vielfärbbarkeit der verschiedenartigen Zellen der Nebenniere stimmen die manchelei Untersuchungsergebnisse über deren feinere Teile nicht immer über, und die Ansichten vieler Autoren weichen weit voneinander ab. Indem Verf. źum Material Meerschweinchen wählte, welche zur funktionellen Untersuchung der Nebenniere als Experimenttier am meisten zur Verfügung stehen, wurde die morphologische Untersuchung über die Nervenelemente der Nebenniere angestellt, und an dieser Stelle sollen einige Befunde mitgeteilt sein.

\section{MATERIAL UND METHODE}

Zum Experimenttier dienten 40 gesunde, reifende Meerschweinchen (今) vom Kör pergewicht $380-580 \mathrm{~g}$. Nachdem sie alle durch den Stich von Medulla oblongata getötet. wurden, wurden 35 Stücke sofort ins $10 \%$ ige Neutralformalin, die andere 5 in den abso- 
luten Alkohol gelegt, und resp. fixiert. 30 Stücke von den im Formalin fixierten wurden in Gefrierschnitte von $30 \mu$ dick zerlegt, und mit Suzukischer Silberimprägnationsmethode und der "Sugamo"-Markscheidenfärbung behandelt. Nachdem die übrigen 5 in Zelloidin eingebettet wurden, wurden sie wiederum in Serienschnitte von $20 \mu$ zerlegt, und darauf wurde Hämatoxylin-Eosin-Färbung angewandt. Um die Nervenzellen der in Alkohol fixierten Exemplaren zu untersuchen, wurden sie in Zelloidin eingebettet und in Schnitte von $10 \mu$ zerlegt. Dann wurde die Nissl'sche Färbung mit der $0.1 \%$ igen Toluidinblauund Thioninflüssigkeit ausgeführt.

\section{BEFUNDE UND ZUSAMMENFASSUNG}

\section{1) INNERVATION DER RINDE}

Ueber die Innervation der Nebennierenrinde finden sich seit Dogiel manchelei Berichte. Die in der Rinde verteilten Nervenelemente sind dem Mark gegenüber ganz spärlich vorhanden, ihre peripherer Teil ist so ausserordentlich fein, wie es erst bei starker Vergrösserung bemerkt wird, und noch dazu lassen sie sich sehr schwer imprägnieren. Infolgedessen weichen die Ergebnisse der Untersuchungen weit voneinander ab. Stöhr, Sato u. a. haben in ihren Arbeiten gute Befunde über die feine Innervation der Rinde mit ihrer ausgezeichneten Färbungsmethode und stärkerer Vergrösserung beschrieben. Aber einige Autoren wie Hoshi, Swinyard u. a. stellten das Vorhandensein der in der Rinde verteilten Nervenfasern offenbar in Abrede, und teilten mit, dass die argyrophilen Fasern in der Rinde nicht anders als die Reticulumfasern sind. Dass der Mangel ihrer Berichte darin besteht, dass die Nervenfasern auf Grund der Unvollständigkeit der Färbungsmethode unfärbar sind, lässt sich leicht vorstellen.

Bei dem vorliegenden Studium hat Verf. die in der Rinde ausgebreiteten Nervenfasern folgendermassen in zwei Gruppen eingeteilt:

1) Erste Gruppe: Nämlich feine marklose Nervenfasern, welche sich unter Begleitung von kleinen Blutgefässen in die Rinde begeben, das Nervengeflecht in der Kapsel der Nebenniere und die aus dem Plexus medullaris stammenden Nervenfasern.

2) Zweite Gruppe: Nervenfasern, welche von der Kapsel durch die Rinde hindurchziehend zum Marke gelangen, und sich auf diese Weise an Bildung des Plexus medullaris beteiligen. Da, wie oben erwähnt, die Neıvenfasern der zweiten Gruppe von der Kapsel her durch die Rinde ohne irgendwelche Astabgabe hindurchziehen, werde ich darum diese Nerven unten im Abschnitte des Markes beschreiben.

Nervenfasern, welche mit der Nervenversorgung der Rinde im unmittelbaren Zusammenhang stehen, sind die der ersten Gruppe, über deren Versorgung sind schon 
manchelei oben angeführte Angaben erstattet worden. Während Dogiel und Stöhr darauf hingewiesen haben, wie ich es in meinen Präparaten gesehen, dass die Nebenniererinde mit den in Begleitung der efferenten und afferenten Gefässe verlaufenden vegetativen Nervenfasern, dem Nervengeflechte in der Kapsel und dem Plexus medullaris innerviert ist, berichteten andererseits einıge Autoren, wie Sato, dass diese Rinde lediglich mit den die kleinen Gefässe und Kapillaren entlang laufenden innerviert sei.

Trotzdem kann ich mich meinem Befunde nach an die jenige Ansicht nicht anschliessen, wonach Nervenfasern, welche von der Kapsel her in die Zona glomerulosa, resp. aus dem Marknervengeflecht in die Zona reticularis hineingelangen, auf die Rinde keinen Einfluss ausüben, und auch mit dieser nichts zu tun haben sollen. (Vgl. $A b b .1,2,3$, )

In der Kapsel der Nebenniere befindet sich das stärkere Nervengeflecht, und darin sind die sich in einzelnen oder in Dutzenden gruppierenden, peripheren sympathischen Ganglienzellen nachzuweisen. Wiederum sind darin die Fülle von marklosen Nervenfasern und kleine Menge von markhaltigen vorhanden, und diese Nervenfasern beide verästeln sich und dringen zum Teil in de Zona glomerulosa ein. Sensible Endorgane in Gestalt von baum- und knopfförmigen Endigungen, die Kura früher beschrieben hat, vermochte Stöhr im Kapselgewebe nicht zu entdecken. Obgleich ich in der Kapsel markhaltige Fasern bemerkte, gelang mir nicht diese baum-und knopfförmigen Endigungen festzustellen.

Die Nervenfasern der Zona glomerulosa stammen aus dem in der Kapsel befindlichen Nervenfasern, und sie zwängen sich unter Verästelung zwischen die Drüsenzellen hinein. (Vgl. $A b b .1$ u. 2)

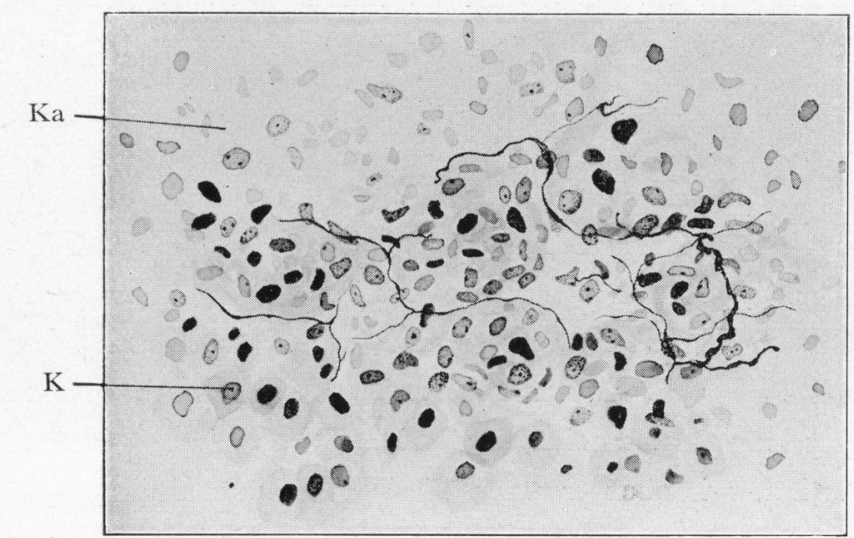

Abb. 1. Innervation in der Zona glomerulosa Ka; Kapsel K; Kern der Rindnzellen in der Zona glomerulosa, $700 \mathrm{mal}$ vergrössert, auf $1 / 2$ verkleinert. 


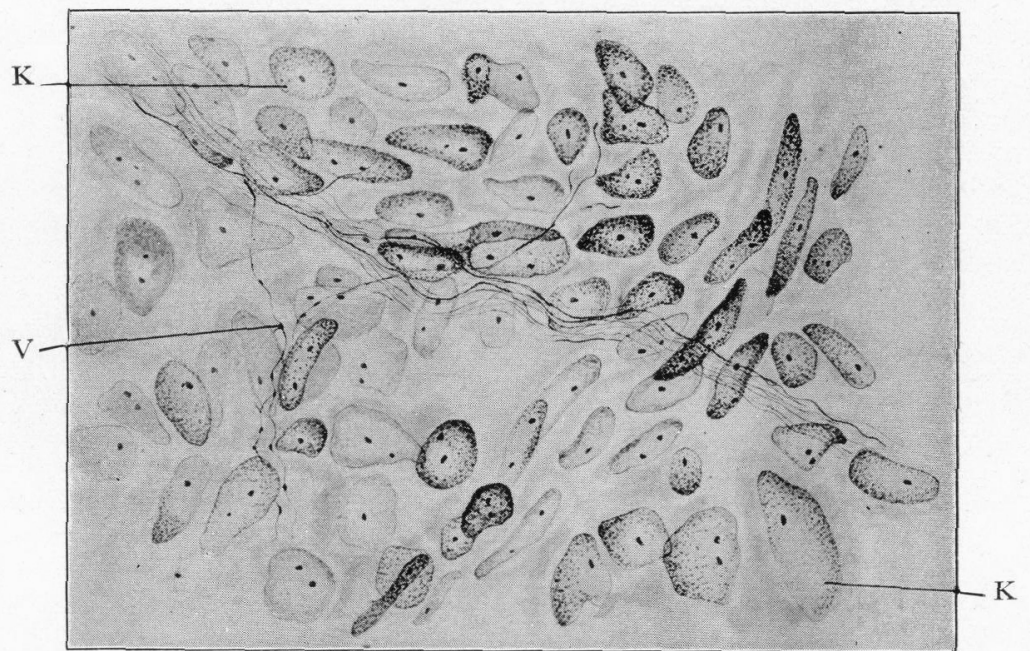

$A b b$. 2. Innervation in der Zona glomerulosa bei starker Vergrösserung. $\mathrm{K}_{1}$; Kern der Rindenzellen in der Zona glomerulosa. $\mathrm{K}_{2}$; Kern der Rindenzellen in der Zona fascicularis. $\mathrm{V}$; Varicöse Anschwellung. 2000 mal vergrössert, auf $1 / 2$ verkleinert.



Abb. 3. Verlauf der Nervenfasern in der Zona fascicularis. C ; Kapillare. Z ; Rindenzellen in der Zona fascicularis. 2000 mal vergrössert, auf $1 / 2$ verkleinert. 
In der Zona fascicularis sind die die kleinen Gefässe und Kapillaren entlang laufenden Nervenfasern oder die sich zwischen den Zellen der Zona fascicularis schlängelnden marklosen Nervenfasern zu finden, und diese beiden verzweigen sich und treten zwischen die Zellen ein. (Vgl. Abb.3)

Die Zona reticularis ist an Nervenmassen erheblich reicher als die Zona glomerulosa und fascicularis, und die vom Marknervengefechte herstammenden Nervenfasern beteiligen sich zusammen mit denjenigen Fasern, welche die von Zona fascicularis herkommenden kleinen Gefässe entlang verlaufen, und mit denjenigen, welche sich zwischen den Zellen der Zona fascicularis schlängeln, an der Innervation der Zona reticularis. (Vgl. Abb. 4)

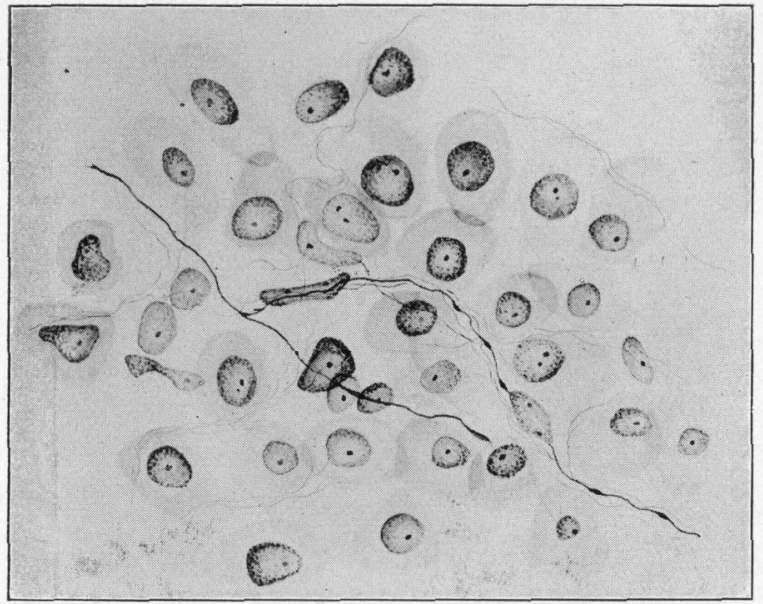

$A b t$. 4. Innervation in der Zona reticularis.

2000 mal vergrössert, auf $1 / 2$ verkleinert.

Wenn man die Innervation der letztgenannten Schichten bei starker Vergrösserung betrachtet, verzweigen sich die Nervenfasern in feire Neurofibrillen, verlaufen an der Zellenoberfäche zwischen den einzelnen Rindenzellen, treten miteinander in Verbindung und dann bilden ein besonderes Terminalretikulum. Obwohl dieser Befund von dem des Stöhr in bezug auf typisches Terminalretikulms etwas verschieden ist, entspricht er fast dem Befund von Sa1o an Menschen.

An den Neurofibrillen zwischen den Rirdenze!len finden sich ebensowenig sogenanntes Schwannsches Leitungsplasmodium, wie Schwann's Kern. Alpert schilderte das typische Terminalretikulum als ein vegetatives Endnetz an den sämtlichen Schichten der Nebennierenrinde. Aber sowohl ich wie Riegel konnten dasselbe nur an der Kapillarenwand der Rindenzellen feststellen. (Vgl. Abb. 5) 




Abb. 5. Typisches Terminalreticulum an der Kapillarenwand in der Zona reticularis. $\mathrm{T}$; Terminalretikulum. C; Kapillare.

$2000 \mathrm{mal}$ vergrössert, auf $1 / 2$ verkleinert.

Während Dogiel, Pines und Narowtschatowa knopfförmige Anschwellung als die Endigungsform der Nerven in der Rinde beschrieben, hat Stöhr sie doch entscheidend verleugnet. Mir scheint vielmehr, dass es sich um eine Nervenendigung, welche mit der Blutgefässwand zu tun hat, handeln möchte, sonst müsste sie nicht anderes als ein Artefakt, welches mit der Silberimprägnationsmethode produziert wurde, zu ersehen.

2) DIE NERVOESEN ELEMENTE DES MARKES

\section{A) NERVENFASERN}

Das Mark ist an Nervenmassen beträchtlich reich, weil es selbst der embryologischen Betrachtung nach aus sympathischen Ganglien herstammt. Das im Marke ausgebildete Plexus medullaris besteht, wie zahlreiche Autoren beschrieben haben, aus folgenden dreiartigen Nervenfasern, d. h. erstens aus den durch die Rinde hindurch ins Mark ziehenden Nervenfasern, zweitens aus den die efferenten und afferenten Blutgefässe entlang verlaufenden, marklosen Nervenfasern an derjenigen Stelle, wo des Mark mit der Kapsel unmittelbar in Berührung steht, und drittens aus den von sympathischen Ganglienzellen des Markes herstammenden Nervenfasern. Es bildet sich auf diese Weise ein kompliziertes, rauhes Nerveanetz, (Vgl. Abb. 6 u. 7) 


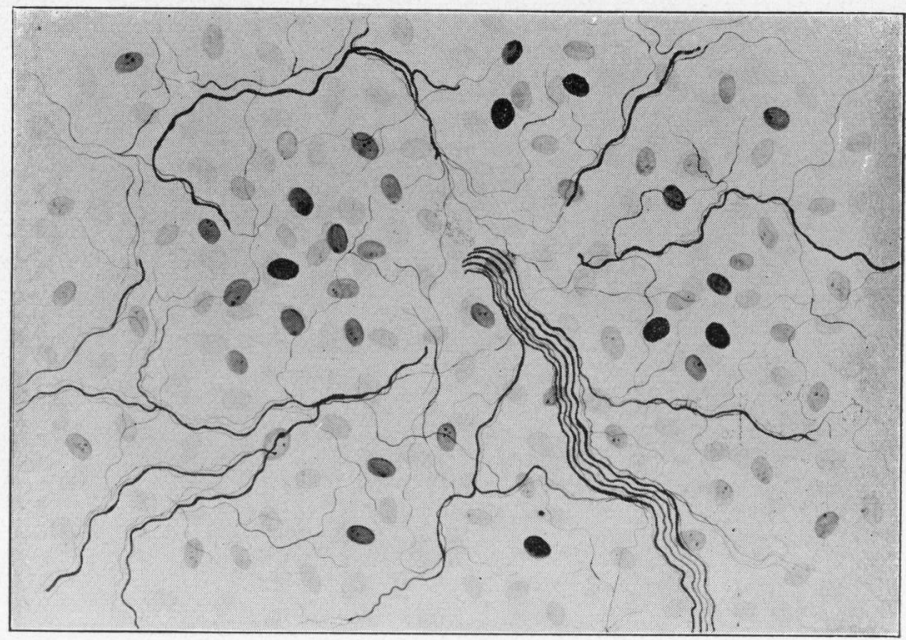

$A b l$. 6. Schema der Nervenversorgung im Markes.

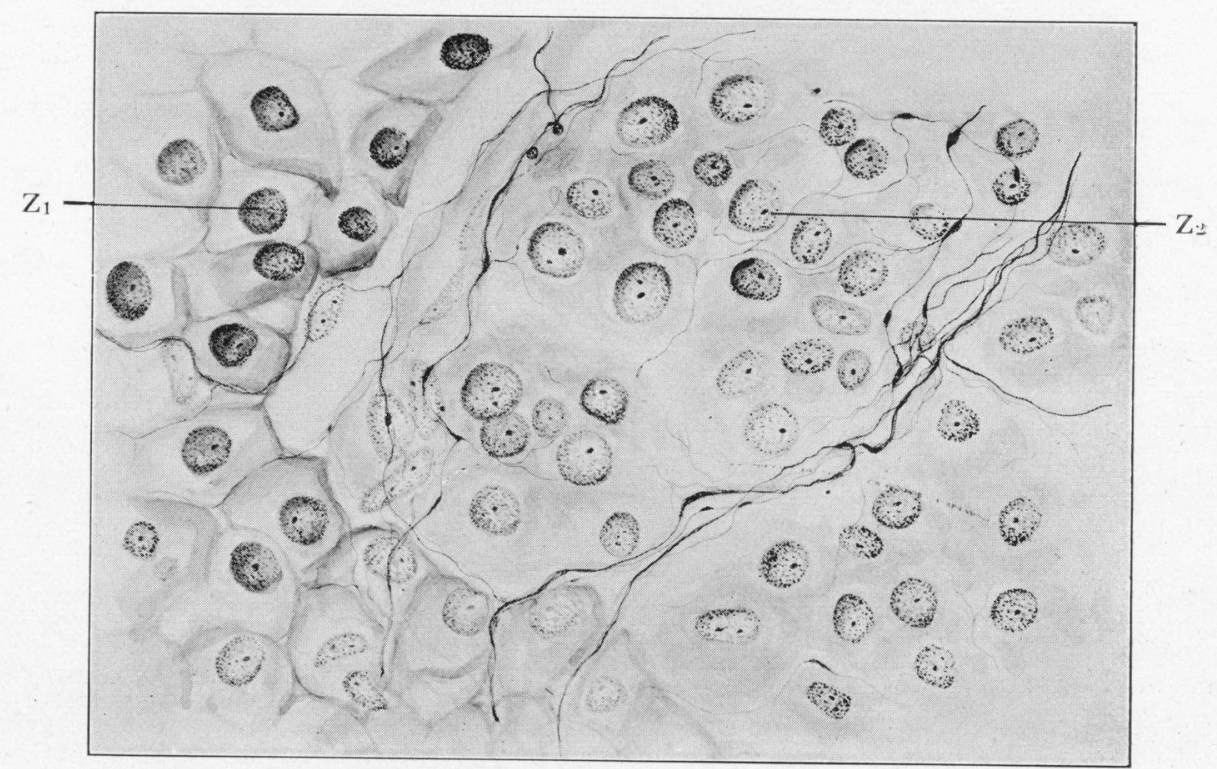

Abb. 7. Innervation im Markes.

$Z_{1}$; Rindenzellen in der Zona reticularis.

$\mathrm{Z}_{2}$; Markzellen.

$2000 \mathrm{mal}$ vergrössert. auf $1 / 6$ verkleinert.

Zum Teil ziehen als Nervenbündel die Nervenfasern aus der Rinde durch die Rinde in einer geraden Linie hindurch, zum Teil dringen sie zusammen mit der einzigen oder 
den einigen Nervenfasern von der Kapsel her in die Rinde ein und schlängeln sich zwischen den Rindenzellen. Auf dem Wege verlieren diese beiden Nerven in der Zona fascicularis, reticularis oder im Marke ihre Markscheide, und die Bündel ändern ihre Richtung und dann splittern sich in weitere Verzweigung auf. Uebrigens ziehen sie beide durch die Rinde hindurch und scheinen mit keinen Rindenzellen im dichten Zusammenhang zu stehen. (Vgl. Photo. 1.u. 2)

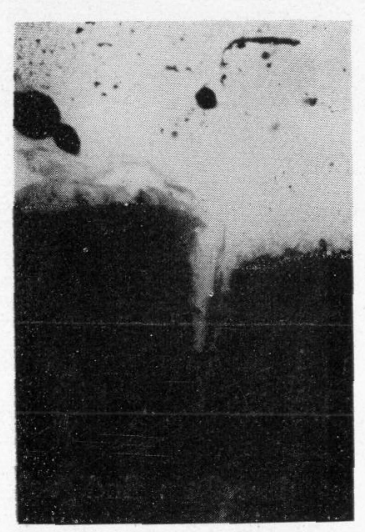

Photo. 1. Aus Kapsel eindringende markhaltige Nervenfasern.

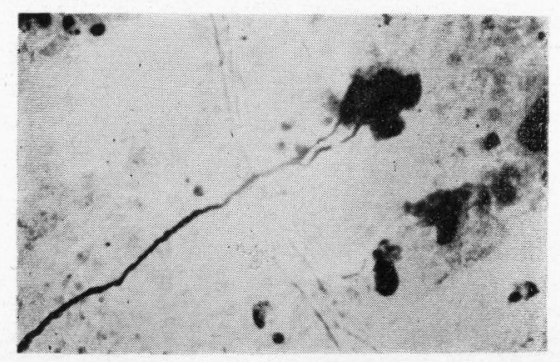

Photo. 2. Im Marke abzweigende markhaltige Nerverfasern.

Auch die unter Begleitung der Gefässe eindringenden Nervenfasern entsprechen der Verlaufsrichtung der Gefässe im Mark, und sich von den Gefässwänden alìmählich abtrennend, bilden sie zusammen mit den aus sympathischen Ganglienzellen herstammenden Nervenfasern das Marknervengeflecht. Das Nervengeflechı im Marke ist ungefähr gleichmässig verteilt und umbüllt die Markzellengruppe. Die Nervenfasern verzweigen sich weiter in feinere Neurofibrillen, dringen zwischen jede Markzellen ein, und diese verschlingend und umklammernd anastomosieren sie miteinander, und dann bilden grobes Nervennetz. (Vgl, $A b b .8$ ) Aus dem Vorhergehenden geht hervor, wie es Sato an Menschen 


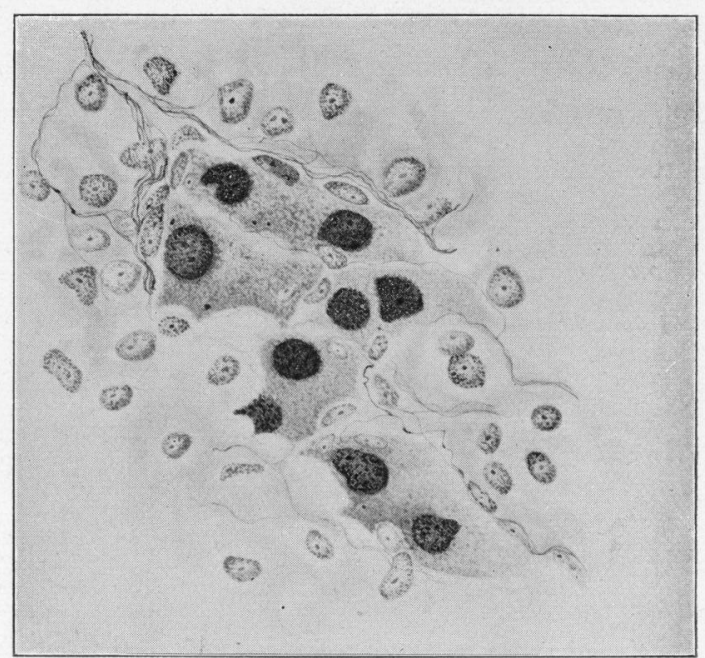

Abb. 8. Form der Ganglienzellen mit der Silberimprägnation (Suzuki'sche Methode). 1300 mal vergrössert, auf $1 / 2$ verkleinert.

berichtet, dass im Mark keine besondere freie Endigung zu finden ist, die Nervenfasern die Markzellen umbüllend, miteinander in kontinuierlicher Verbindung stehen und meistens in Gestalt eines groben Netzes ein verschlossenes Nervennetz dicht an der Oberfl̈̈che der Markzellen entwickeln.

Obwohl einerseits Dogiel, Kolossow, Kura, Watanabe, Pines und Narowtschatowa u. a. eine knoten-, oder plattenartige Endverdickung beschrieben und sie als Nervenendigung bezeichnet haben, konnten andererseits Stöhr und Sato solch eine Bildung nirgends nachweisen. Ueber die Möglichkeit ihres Vorhandseins weichen daher verschiedene Ansichte voneinander ab. Meiner Betrachtung nach ist im Marke eine knotenförmige Anschwellung, welche diesen Endkolben zu entsprechen scheint, nachzuweisen. Aber nach dem Silberimprägnationszustand und seinem Verhalten mit den Blutgefässen betrachtet, handelt es sich nicht um eine Nervenendigung, welche auf die Markzellen funktionelle Wirkung ausüben. Sensible Nervenendigungen vom charakteristis chen Bau hat Stöhr nicht gefunden, und ebenfalls habe ich leider relativ komplizierte knäuelartige Endigungen, die Sato vom Mark beim Menschen abgebildet, nicht entdecken können.

\section{B) GANGLIENZELLEN}

Ueber die Innervation des Markes finden sich manchelei Berichte, darin lassen sich vorwiegend die Anordnung und die Form der Ganglienzellen mit der Imprägnationsmethode darstellen, während bei zytologischer Untersuchung, wie sie Enjo(4) ausführte, Nissl'sche Färbung in Nervenzellen (Mensch) zu verfolgen ist. Zum Studium dieser 
Nervenzellen hat Verf. zusammen mit der Silberimprägnationsmethode sowohl Nissl'sche Färbung als auch Hämatoxylin-Eosin-Färbung angewandt und folgende Resultate erhalten. Die Ganglienzellen liegen teils vereinzelt, teils in kleinen Gruppen oder zu grösseren Ganglien angehäuft wahllos über die ganze Markschicht hin verstreut, und sie sind vor allem an der Grenzstelle zwischen Mark und Rinde relativ zahlreich nachzuweisen. Die Zellen sind, wie Ito ${ }^{(7)}$ behauptet, mit der Eigentümlichkeit der allgemeinen, peripheren und autonomen Nervenzellen versehen, und ihre Gestalt und Grösse sind zwar verschiedenartig, aber alle multipolar. Im Zellkörper ist gewöhnlich der mononukuläre, rundförmige Kern exzentrisch vorhanden, of $\mathrm{t}$ zu finden sind zwei, selten ennige Kerne ( $3 \mathrm{u} .4)$. Die Ganglienzellen sind mit von Kohn ${ }^{(8)}$, Penitschka ${ }^{(13)}$ und Stöhr ${ }^{(19)}$ beschriebenen Neben-oder Mantelzellen umgeben. Sie liegen, wie in Abb. 8 gezeigt wird, dicht an der Oberfläche der Ganglienzellen, und deren äussere Oberfäche ist mit den schwachen Bindegeweben umgeben. Darin lassen sich die von Stöhr und Sato erwähnten kolben-oder schlingenförmige, nervöse Endigung pericellulares Nervengeflecht bemerken. (Vgl. Abb. 9)

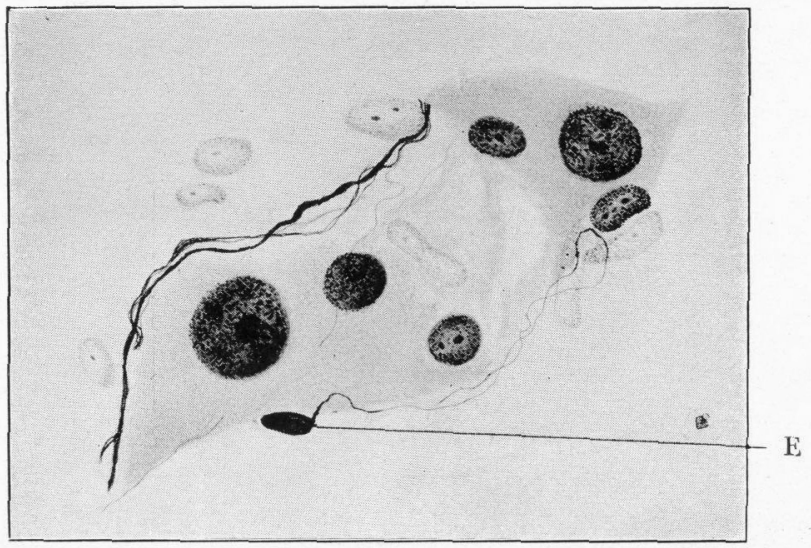

Abb. 9. Einzelne Ganglienzellen mit Silberimprägnation. (Suzukische Methode).

E; Endokolben

2000 mal vergrössert, auf $1 / 2$ verkleinert.

Nach kurzem Verlauf auf der Oberläche der Ganglienzellen gelangen die in Abb. 9 dargestellten Nervenfasein zum Zellenkörper, und bilden an diesem Teile oder im umgebenden Bindegewebe das Endapparat der Endokolben und der schlingenförmigen Bildung. Zwar nimmt Stöhr an, dass der Endapparat aus den sensiblen Endigungen herstammt, und Sato behauptet, dass dieses sich aus kurzem Fortsatz der Ganglienzellen entwickelt, aber an meinen Präparatea lässt sich die Herkunft noch nicht klar machen.

Nisslsche Körperchen sind als feine granulierende in den Ganglienzellen diffus 
verteilt, und darin ist kein typisches Tigroid zu finden. (Vgl. $A b b .10$ )



Abb. A. 2000 mal vergrössert, auf $1 / 2$ verkleinert.

$A b b . B, 1300 \mathrm{mal}$ vergrössert, auf $1 / 2$ verkleinert.

Abb. 10. Form der Ganglienzellen durch die Nissl'schen Färbung.

Nach der Versorgungsweise der Nissl'schen Körperchen teilte En jo die Ganglienzellen folgendermassen ein:

Der 1. Typ: Nissl'sche Körperchen sind in den Zellen gleichmässig versorgt.

Der 2. Typ: Feine granulierende Nissl'sche Körperchen versammeln sich in der Umgebung der Zellen. (peripherer Ring).

Der 3. Typ: Nissl'sche Körperchen versammeln sich vorzugsweise um den Kern. (Perinuklearer Ring)

Der 4. Typ: Nissl'sche Körperchen bilden den peripheren Ring und den perinuklearen.

Der 5. Typ: Nissl'sche Köıperchen bilden an der unbestimmten Stelle der Zellen unregelmässige Masse.

An meinen Präparaten sind die oben genannten Typen der Ganglienzellen deutlich nachzuweisen, indem der 2. Typ am häufigsten, der 2, u. 3. häufiger und der 4, u. 5 . selten zu finden sind. Aber auf Grund der Versorgung der Nissl'schen Körperchen kann man zwischen Neurit und Dendrit sehr schwer unterscheiden.

\section{SCHLUSS}

Ueber die Nebenniere der reifenden Meerschweinchen habe ich die Innervation mit der Suzuki'schen Silberimprägnationsmethode, "Sugamo"-Markscheidenfärbung, Nissl' schen Färbung und Hämatoxylin-Ecsin-Fürbung untersucht und folgende Ergebnisse erhalten. 


\section{1) INNERVATION DER RINDE}

Dem Mark gegenüber sind die Nervenelemente der Rinde ganz spärlich zu finde n, die aus der Rinde herstammenden oder kleine Gefüsse begleitenden marklosen Nerven verzweigen sich zwischen den Rindenzellen, und sie stehen über die sümtlichen Schich te hin mit jeder Rindenzelle in Berühıung, ohne in die Drüsenzellen einzudringen.

Das ist zwar vom typischen Terminalretikulum,wie Stöhr es beschreibt, etwas verschieden, aber es handelt sich um ein besonderes Terminalretikulum.

\section{2) INNERVATION DES MARKES}

\section{A) NERVENFASERN}

Die Nervenfasern ziehen teils als Nervenbündel einschliesslich der markhaltigen Nerven durch die Rinde geradlinig hindurch, und teils zusammen mit der einzigen oder den einigen Nervenfasern dringen sie von der Kapsel her in die Rinde ein und schlängeln sich zwischen den Rindenzellen. Auf dem Wege verlieren diese beiden Nerven in der Zona fascicularis, reticularis oder im Marke ihre Markscheide, und die Bündel ändern ihre Richtung und dann splittern sich in weiter Verzweigung auf. Die die Blutgefässe im Marke begleitenden Nervenfasern und die von Markganglienzellen herstammenden vereinigen sich zu einem komplizierten Marknervengeflecht. Die Nervenfasern verästeln sich weiter und umhüllen jede Markzellen. Nach der Anastomosierung miteinander bilden die Neurofibrillen ein grobes Nervennetz. An einer Stelle des Markes aber lassen sich zweilen knotenartige Anschwellungen bemerken.

\section{B ) GANGLIENZELLEN}

Die Nervenzellen liegen teils vereinzelt, teils in kleinen Gruppen oder zu grösseren Ganglien angehäuft wahllos über die ganze Markschichte hin verstreut, und sie sind an der Grenzstelle zwischen Mark und Rinde relatıv reich nachzuweisen. Jede Nervenzellen sind resp. mit dem Charakter der typischen, peripheren und autonomen Nervenzellen versehen. Nach der Einteilung Enjos auf Grund der Versorgung der Nissl'schen Körperchen ist der zweite Typus am häufigsten zu finden, und der erste zunärhst dem zweiten häufiger. In Nervenzellen ist im allgemeinen kein typischer Tigooid zu bemerken. Mit der Silberimprägnationsmethode sind in der Umgebung der Zellenkörper Nebenund Mantelzellen nachzuweisen, und die aüssere Oberfläche ist mit dem schwachen Bindegewebe umgeben.

In diesen Bindegeweben ist ein perizellulär es Nervengeflecht, und dicht an der Oberfläche der Zellen oder in deren Nähe kolbenartiger oder ringförmiger Endapparat zu finden. 


\section{LITERATUR}

1. Alpert, L.K.: The innervation of the suprarenal glands. Anat. Rec. 50, 221-233, 1931 .

2. Dogiel, A. S.: Die Nervenendigungen in den Nebennieren der Säugetiere. Arch. Anat., Anat. Abt. 90-104 1894,

3. Dostojewsky, A.: Ein Beitrag zur mikroskopischen Anatomie der Nebennieren bei Säugetieren. Arch. mikrosk. Anat. 27, 277-296, 1886.

4. ENJO, K.: Cytologische Untersuchungen der Ganglienzellen im Nebennierenmarke der Menschen. Jossi Igaku Kenkyu. Vol. 13. No. 3. 251-256, 1943. (Japanisch).

5. Hirt, Augustzur.: Innervation der Niere und Nebenniere des Frosches. Z. Anat. u. Entw. gesch. $91,580-593,1930$.

6. HOSHI, TOGO: Histology of nerves in the suprarenals. Tohoku Igaku. Zasshi 9, 4-5 $1926 \mathrm{~b}$.

7. ITO. u. KUBO: Zytologische Untersuchungen über die intramuralen Ganglienzellen des Verdauungstractus. Ueber die Ganglienzellen des menschlichen Darms, mit besonderer Berïcksichtigung auf die Nisslsubstanz. Cytologia. Bd. 10. 334, 1940.

8. Kонn.: Ueber die Scheidenzellen peripheren Ganglienzellen. Anat. Anz. 30. 154, 1907.

9. Kонno, Shigenoba. Zur vergleichenden Histologie und Embryologie der Nebennieren der Säuger und Menschen. Z. Anat. 77, 419-430, 1925.

10. Kolossow, N.G.: Zur Frage der Innervation der Nebennieren. Z. mikrosk. anat. Forschg. 20, 107, 1930.

11. KurA, N.: Ueber die Innervation der Nebennieren. Mitt. med. Akad.Kioto. 1, 107-124, 1927. (Japanisch)

12. Moellendorff, W. u. Bargmann W.: Die Nebenniere. Neurosekretion. Handbuch der Mikroskopischen Anatomie des Menschen, 1954.

13. Penitschka.: Ueber den Bau des Ganglion cervicale uteri des Menschen, Beriicksichtigung der mehr kernigen Ganglienzellen und des chromaffinen Gewebes. Anat. Anz. 66, 417, 1929.

14. Pines, J. L. Ja., u. Narowtschatowa.: Ueber die Innervation der Nebenniere. Z. mikrosk. -ant. Forsch. 25, 518, 1931 .

15. Renner, O.: Die Innervation der Nebenniere. Dtsch. Arch. Klin. Med. 114, 473-482, 1914.

16. Riegele, L.: Die Bedeutung des reticuloendothelialen Syncytiums als Scheidenplasmodium des fibrillären nervösen Endnetzes in Leber, Milz und Nebennieren Z. Zellforsch. 15, 311-330, 1932.

17. SATO, A.: Innervation of Corpus Suprarenale in Human Adult. Tohoku J. Exper. Med. 55, 259$271,1952$.

18. Stoehrjr., Philipp.: Zur Innervation der menschlichen Nebennieren. Z. Znat. 104, 475-490, 1935.

19. StokнR: Ueber Nebenzellen und deren Inmervation in Ganglien des vegetativen Nervensystems zugleich ein Beitrag zur Synapsenfrage. Z. Zellforsch. Abt. A. 29, 569, 1939.

20. Swinyard, C. A.: The innervation of the suprarenal glands. Anat. Rec. 68, 417-429, 1937. 
21. Watanabe u. Mimura: Nervous termination of the suprarenal gland. Kaibogaku Zassi (Acta Anatomica Nipponica) Vo 129. No 2, 1954. (Japanisch). 